\title{
CORRIGENDUM
}

\section{Bottlenecks, population differentiation and apparent selection at microsatellite loci in Australian Drosophila buzzatii}

JSF Barker, J Frydenberg, J González, HI Davies, A Ruiz, JG Sørensen and V Loeschcke

Heredity (2009) 102, 626; doi:10.1038/hdy.2009.43

Correction to: Heredity (2009) 102, 389-401; doi:10.1038/ sj.hdy.2008.127

The authors have realized that an incorrect version of Table 3 was published.

Table 3 Linkage disequilibrium analyses

\begin{tabular}{|c|c|c|}
\hline Population & Mean $D^{\prime}$ & $\begin{array}{c}\% D^{\prime} \text { significant } \\
\mathrm{P}<0.05\end{array}$ \\
\hline MUB & 0.303 & 7.62 \\
\hline ISG & 0.270 & 3.81 \\
\hline GRD & 0.260 & 6.67 \\
\hline HIS & 0.255 & 2.86 \\
\hline BAR & 0.298 & 15.24 \\
\hline TAM & 0.260 & 6.67 \\
\hline GER & 0.206 & 3.81 \\
\hline MAL & 0.239 & 7.62 \\
\hline BUL & 0.250 & 1.90 \\
\hline OTA & 0.439 & 3.81 \\
\hline CAT & 0.485 & 4.76 \\
\hline \multicolumn{3}{|c|}{ (b) Number of $D^{\prime}$ tests that were significant } \\
\hline $\begin{array}{l}\text { For loci on the same } \\
\text { chromosome }\end{array}$ & $\begin{array}{l}\text { For loci on different } \\
\text { chromosomes }\end{array}$ & $\begin{array}{c}\text { Unknown } \\
\text { (involve Db003) }\end{array}$ \\
\hline \multicolumn{3}{|l|}{ Australian populations } \\
\hline Chromosome 2-23 & 2 and $4-11$ & Chromosome 2-5 \\
\hline Chromosome 4-7 & 2 and $5-6$ & Chromosome 4-1 \\
\hline Chromosome 5-1 & 4 and $5-4$ & Chromosome $5-1$ \\
\hline \multicolumn{3}{|l|}{ Argentine populations } \\
\hline Chromosome 2-1 & $\begin{array}{l}2 \text { and } 4-6 \\
2 \text { and } 5-1\end{array}$ & \\
\hline
\end{tabular}

Please see the correct table below.

The authors apologize for this error. 